\title{
High mortality of CKD patients on hemodialysis with Covid-19 in Brazil
}

\author{
Andrea Pio-Abreu ${ }^{1}\left[\right.$ - Marcelo Mazza do Nascimento ${ }^{2} \odot \cdot$. Marcos Alexandre Vieira ${ }^{3}$. \\ Precil Diego Miranda de Menezes Neves ${ }^{1} \cdot$ Jocemir Ronaldo Lugon $^{4}$ (D) $\cdot$ Ricardo Sesso $^{5}$ (i)
}

Published online: 8 August 2020

(C) Italian Society of Nephrology 2020

The first case of the novel coronavirus disease (Covid-19) pandemic in Brazil was reported on February 26th, 2020, in São Paulo. On June 30th, Brazil ranked 2nd in the world in the number of cases, with 1.4 million victims officially reported and an average number of 37,600 new cases per day [Coronavirus disease case panel 2019 (COVID-19) in Brazil by the Ministry of Health. Available at: https://covid.saude .gov.br/. Accessed June 27, 2020.].

Brazil is the third country in the world in the number of patients on chronic dialysis, with 133,500 in July, 2018 [2]. Ninety-two percent of them are on in-center hemodialysis (HD). A large number of kidney disease patients particularly those with kidney failure on dialysis are at a higher risk of complications of severe acute respiratory syndrome coronavirus 2 (SARS CoV-2) infection [3, 4].

The Brazilian Society of Nephrology, in conjunction with the Brazilian Association of Dialysis and Transplant Centers, has been conducting a national on-line survey of Covid-19 on kidney failure patients undergoing regular HD, since June 1st, 2020. In the present study, we describe the incidence, mortality, and fatality rates due to Covid-19 since the beginning of the outbreak until June 30th in a sample of 37,852 hemodialysis patients.

Cases were considered confirmed if they had laboratory isolation of the SARS-CoV-2 by RT-PCR test from nasopharyngeal/oropharyngeal swabs.

As of June 30th, there were 1,402,041 cases and 59,594 deaths reported in the overall Brazilian population $[1,5]$.

Andrea Pio-Abreu

andreapioabreu@gmail.com

1 Nephrology Division, School of Medicine, Universidade de São Paulo, São Paulo, SP, Brazil

2 Universidade Federal do Paraná, Curitiba, PR, Brazil

3 Associação Brasileira dos Centros de Dialise e Transplante, Brasília, Brazil

4 Universidade Federal Fluminense, Niteroi, RJ, Brazil

5 Universidade Federal de São Paulo, São Paulo, SP, Brazil
Two hundred and seven dialysis centers, representing $26 \%$ of the 805 Brazilian centers responded to the survey. These centers derived from 24 of the 27 Brazilian states and their distribution by region was similar to that in all the Brazilian states (6\% North, $16 \%$ Northeast, $8 \%$ Midwest, 51\% Southeast, and $19 \%$ South). Likewise, the nature of the centers (73\% private, and $27 \%$ public) and the predominant funding by the Brazilian public health system (80\%) instead of private (20\%) were similar to those of general centers in the country [2].

The total number of HD patients in the study centers was 37,852 . Of these, 1291 were confirmed for Covid-19, and 357 died. The incidence, mortality, and fatality rates in HD patients were $341 / 10,000$ patients, $94 / 10,000$ patients, and $27.7 \%$, respectively (Table 1). The number of Covid-19 patients, their incidence, and mortality rates in the general Brazilian population are also shown in Table 1. The incidence, mortality, and fatality rates in the HD population were remarkably higher than the general population reaching 5.1, 33.4, and 6.4 times higher, respectively. Incidence and mortality rates in the HD patients varied widely by region, being higher in the North (Amazon region-states of Pará and Rondônia), Northeast (states of Paraíba and Pernambuco), and Southeast regions (Table 1).

Covid-19 suspected and confirmed patients were dialyzed in a separate room in $78 \%$ of the centers. In $54 \%$ of the centers, they were switched for an exclusive dialysis shift. The collection of specimens for Covid-19 diagnosis was carried out on-site in $27 \%$ of the dialysis centers: nasopharyngeal swabs for RT-PCR in 17\%, and blood for antibody detection in $18 \%$ of the centers.

As for the dialysis staff members working in these centers, Covid-19 was confirmed or suspected (individuals with suggestive symptoms of the disease such as fever, cough, dyspnea or asthenia/myalgia, but without a diagnostic RT-PCR test performed) in $11.1 \%(n=113 / 1014)$ of the nephrologists, $11.3 \%(n=80 / 707)$ of the nurses and $11.9 \%(n=495 / 4163)$ of the dialysis technicians. None of 
Table 1 Cases of Covid-19, incidence, mortality, and fatality rates in the Brazilian population and the hemodialysis (HD) population

\begin{tabular}{|c|c|}
\hline Overall Brazilian population, $n$ & $211,528,297$ \\
\hline Covid-19 cases, $n$ & $1,402,041$ \\
\hline Covid-19 deaths, $n$ & 59,594 \\
\hline Incidence rate $/ 10,000^{\mathrm{a}}$ & 66.7 \\
\hline Mortality rate $/ 10,000^{\mathrm{a}}$ & 2.8 \\
\hline Fatality rate, $\%$ & 4.3 \\
\hline Brazilian HD population, $n$ & 130,192 \\
\hline Participants, $n$ & 37,852 \\
\hline Covid-19 cases, $n$ & 1,291 \\
\hline Covid-19 deaths, $n$ & 357 \\
\hline Incidence rate $/ 10,000^{\mathrm{b}}$ & 341.0 \\
\hline Mortality rate $/ 10,000^{\mathrm{b}}$ & 94.3 \\
\hline Fatality rate, $\%$ & 27.7 \\
\hline \multicolumn{2}{|l|}{ Estimates in HD patients by region } \\
\hline \multicolumn{2}{|l|}{ North } \\
\hline Incidence rate/10,000 & 762.2 \\
\hline Mortality rate/10,000 & 316.3 \\
\hline Fatality rate, $\%$ & 40.5 \\
\hline \multicolumn{2}{|l|}{ Northeast } \\
\hline Incidence rate/10,000 & 489.7 \\
\hline Mortality rate/10,000 & 102.8 \\
\hline Fatality rate, $\%$ & 21.0 \\
\hline \multicolumn{2}{|l|}{ Midwest } \\
\hline Incidence rate/10,000 & 261.9 \\
\hline Mortality rate/10,000 & 83.5 \\
\hline Fatality rate, $\%$ & 31.9 \\
\hline \multicolumn{2}{|l|}{ Southeast } \\
\hline Incidence rate/10,000 & 315.5 \\
\hline Mortality rate/10,000 & 90.1 \\
\hline Fatality rate, $\%$ & 28.5 \\
\hline \multicolumn{2}{|l|}{ South } \\
\hline Incidence rate/10,000 & 90.9 \\
\hline Mortality rate/10,000 & 19.4 \\
\hline Fatality rate, $\%$ & 21.3 \\
\hline
\end{tabular}

Calculations: Incidence rate $=$ the number of cases from $2 / 26 / 2020$ until the end of the current report (6/27/2020) / number of exposed people. Mortality rate $=$ the number of deaths due to Covid-19 / number of exposed people. Fatality rate $=$ (number of confirmed deaths due to Covid-19 / number of Covid-19 cases)*100

The denominator for calculation is ${ }^{a}$ the Brazilian population, and ${ }^{b}$ the studied HD sample

the nephrologists or nurses died but 2 dialysis technicians thus far have.

The present study is the largest survey of Covid-19 incidence and mortality in chronic HD patients reported to date. After 4 months of the first reported case, the pandemic is not under control in the country and the number of cases continues to soar. The outbreak initially affected people from higher social classes and afterward those living in poorer metropolitan areas, thus leading to an overburden in the public health system, including assistance to renal failure patients. Findings of higher mortality and fatality rates in HD patients compared to the general population can be accounted for by their higher mean age, a higher frequency and severity of comorbidities, and less efficient immunological response [6, 7]. Besides, the percentage of testing delivered to this population is conceivably higher than in the general population. As a matter of fact, specimens for Covid-19 tests were collected on-site in $27 \%$ of the centers, thus easing and speeding up the diagnosis workup.

Previous reports on kidney failure patients are restricted to single center data with a small number of patients, but already suggest a more severe form of the disease in this setting [3, 4, 8]. Even considering the effort of some centers to collect specimens for the diagnosis of patients on-site, our rates were possibly affected by the under-testing of infected patients with mild or no symptoms and by non-confirmed Covid-19-related deaths. Therefore, present numbers can be underestimating the incidence and mortality rates and overestimating the fatality rate. Other limitations of this report are the grouped data collection and the voluntary participation of a sample of all Brazilian centers. However, the regional distribution of the study centers was similar to the overall centers in the country including those of the far Amazon and the Northeast regions, which are less economically developed. Also, the funding of the dialysis treatment of the participants accompanied the profile of the whole Brazilian dialysis population. Therefore, although a strict generalization of the results cannot be assured, considering the characteristics of the centers assessed the estimates are conceivably close to the overall situation of the HD patients in the country.

The scenario of the pandemic in the Brazilian population and kidney failure patients on renal replacement therapy unveils the difficulties to prevent the spread of contagious diseases and their complications in a developing country of such large size. Brazil still faces outstanding social inequalities, with many people living in unsafe conditions, without easy access to adequate health care and basic sanitary supply. This is particularly important considering the more fragile health status of dialysis patients.

The present study reports the high mortality rate of Covid-19 in chronic HD patients and reinforces the need for strict preventive measures. These include the early detection of cases and contact tracing, the isolation of patients in the dialysis units, the use of facemasks, the preservation of personal distancing when commuting to the center, and the provision of access to serologic and viral testing. Such precautions should also comprise households.

Acknowledgements The authors acknowledge the valuable contribution of the participating dialysis centers in the survey. 
Data availability Availability of the data at the site: http//:https://abcdt .b2fly.xyz/resultado.

\section{Compliance with ethical standards}

Conflict of interest On behalf of all authors, the corresponding author states that there is no conflict of interest.

Ethical approval This study was approved by the Ethics Committee of the Federal University of São Paulo and was performed in accordance with the ethical standards as laid down in the 1964 Helsinki Declaration.

\section{References}

1. Coronavirus disease case panel 2019 (COVID-19) in Brazil by the Ministry of Health. Available at: https://covid.saude.gov.br/. Accessed June 27, 2020.

2. Neves PMM, Sesso RC, Thomé FS, Lugon JR, Nascimento MM. Brazilian Dialysis Census: analysis of data from the 2009-2018 decade. Braz J Nephrol. 2020. (Ahead of print.)
3. Cheng Y, Luo R, Wang K et al (2020) Kidney disease is associated with in-hospital death of patients with COVID-19. Kidney Int 97:829-838

4. Valeri AM, Robbins-Juarez SY, Stevens JS et al (2020) Presentation and Outcomes of Patients with ESKD and COVID-19. Published online May, J Am Soc Nephrol

5. Brazilian Institute of Geography and Statistics IBGE: Population Estimates, 2019. https://www.ibge.gov.br/estatisticas/socia is/populacao.html. Accessed June 24, 2020.

6. Girndt M, Sester M, Sester U et al (2001) Molecular aspects of T- and B-cell function in uremia. Kidney Int Suppl 78:S206-S211

7. Vaziri ND, Pahl MV, Crum A, Norris K (2012) Effect of uremia on structure and function of immune system. J Ren Nutr 22:149-156

8. Alberici F, Delbarba E, Manenti C et al (2020) Management of patients on dialysis and with kidney transplant during SARSCOV-2 (COVID-19) Pandemic in Brescia. Italy Kidney Int Rep $5: 580-585$

Publisher's Note Springer Nature remains neutral with regard to jurisdictional claims in published maps and institutional affiliations. 